\title{
Is there a new place for mitoxantrone in the treatment of multiple sclerosis?
}

\author{
Sławomir Wawrzyniak, Łukasz Rzepiński \\ Department of Neurology, 10th Military Research Hospital and Polyclinic, Bydgoszcz, Poland
}

\section{ABSTRACT}

Aim of the study. To compare the clinical and neuroradiological efficacy of mitoxantrone (MTX) in various forms of multiple sclerosis (MS), to ascertain whether there is a new place for the drug in the treatment regimen of the disease, as well as to determine its safety profile.

Clinical rationale for the study. Due to the increasing availability of new immunomodulatory therapies in multiple sclerosis (MS), there is a strong need to re-identify clinical variants and stages of the disease in which mitoxantrone (MTX) can be the most effective form of treatment.

Materials and methods. This was a retrospective, non-randomised, observational study evaluating a cohort of $100 \mathrm{MS}$ patients (36 relapsing-remitting - RRMS, 36 secondary progressive - SPMS, and 28 primary progressive - PPMS). 59\% of the RRMS patients had discontinued immunomodulatory therapies (IMTs) within the two years preceding MTX infusion. Patients' disability levels, based on the Kurtzke Expanded Disability Status Scale (EDSS) as well as haematological and echocardiographic parameters, were assessed at baseline and before every infusion. Magnetic resonance imaging (MRI) were performed at entry and after termination of treatment.

Results. We observed a decrease in the median EDSS score from 4.0 at baseline to 3.5 at the end of MTX infusion in the RRMS subgroup, an increase from 4.5 to 5.25 in the PPMS subgroup, and a stable value of 5 points in the SPMS subgroup $(p<0.0001$ ). During the treatment period, $97 \%$ of patients with initial RRMS were free of exacerbations. The baseline EDSS in the RRMS subgroup, as well as the ineffectiveness of previous IMTs, suggested the beginning of conversion to SPMS. We found an $86 \%$ decrease in the proportion of patients with gadolinium-enhancing lesions on MRI after MTX infusions. There were no lifethreatening adverse events of MTX during the period of evaluation.

Conclusions and clinical implications. Mitoxantrone can be considered as a valuable therapeutic option for patients who are on the borderline of RRMS and SPMS.

Key words: mitoxantrone, efficacy, relapsing-remitting multiple sclerosis, secondary progressive multiple sclerosis, disability (Neurol Neurochir Pol 2020; 54 (1):54-61)

\section{Introduction}

Multiple sclerosis (MS) is a chronic, demyelinating disease of the central nervous system with different patterns of evolution and disability accumulation. In the vast majority of patients (85-90\%), the clinical course starts with a relapsingremitting phase (RRMS) that is followed by a secondary progressive phase (SPMS) in more than half of untreated subjects [1-4]. Approximately $10-15 \%$ of patients experience gradually worsening neurological deficits from the onset without clinical relapses: this is known as primary progressive MS (PPMS) [5, 6]. Mitoxantrone (MTX) is one of the most commonly used immunosuppressive drugs in the treatment of MS. MTX is a synthetic anthracenedione derivative of doxorubicin, and was initially used as an antineoplastic agent. Its mechanism of action is through intercalation into DNA strands and inhibition of topoisomerase II enzyme. In this way, the drug causes strand breaks that can delay cell-cycle progression. MTX 
reduces $\mathrm{T}$ lymphocyte proliferation cell numbers, inhibits humoral immunity, and decreases cytotoxic T-cell activity and the secretion of TH1 cytokines [7-9].

In 2000, MTX was approved by the US Food and Drug Administration (FDA) for the treatment of aggressive RRMS, SPMS and progressive-relapsing MS [10]. Thus, the drug can be used both in the induction and escalation treatment approaches in patients with MS, and remains one of the few agents approved for the treatment of SPMS [11]. However, currently the European Medicines Agency (EMA) recommends the use of MTX in highly active RRMS with rapid disability progression in patients for whom alternative treatments are not available [12]. Mitoxantrone can also be considered as a non-selective form of immune reconstitution therapy for very active MS, especially in regions with limited access to newer immunotherapies $[13,14]$. The MTX cumulative lifetime dose has been limited by cardiotoxicity and therapy-related acute leukemia (TRAL), initially to $140 \mathrm{mg} / \mathrm{m}^{2}$ and then to $72 \mathrm{mg} /$ $\mathrm{m}^{2}$ body surface area $[12,15,16]$.

\section{Clinical rationale for the study}

Due to the increasing availability of new immunomodulatory therapies and potentially life-threatening adverse effects of MTX, the use of this drug in the treatment of MS has been significantly reduced. For this reason, there is a strong need to re-identify clinical variants and the stages of multiple sclerosis in which MTX can be the most effective form of treatment.

\section{Materials and methods}

This study was approved by the Committee of Bioethics of Ludvik Rydygier Collegium Medicum (KB 474/2014).

\section{Patient enrolment and clinical evaluation}

We conducted a retrospective, non-randomised, observational study to assess a cohort of 100 Caucasian patients with a diagnosis of MS treated with MTX at the Department of Neurology between July 2002 and September 2014. Eligible patients were aged 18-70 years, fulfilled the diagnostic criteria of multiple sclerosis, had at least one year of documented disease duration, and had disability assessed as being from 2 to 7 on the Kurtzke Expanded Disability Status Scale (EDSS) [17]. Patients with previous immunosuppressive therapy, left ventricular ejection fraction (LVEF) measured by echocardiography of less than $50 \%$, liver or renal impairment, cancer history, HIV infection, or who were pregnant, as well as patients who had undergone intensive steroid therapy during the previous month, were excluded from mitoxantrone treatment. Disease onset was defined as the year of the first symptom. The diagnosis was established according to Poser's criteria [18], McDonald's criteria [19], and Polman et al's revisions to McDonald's criteria [20,21]. The course of the disease was classified as relapsing-remitting (RRMS), or secondary progressive (SPMS), or primary progressive (PPMS) $[1,2]$.

RRMS was defined as the occurrence of disease relapses followed by a complete or partial recovery without progression of disability between the bouts. Relapse was defined as the occurrence of new, or the worsening of previously described, MS-related symptoms lasting more than $24 \mathrm{~h}$ in the absence of infection or fever.

The course of multiple sclerosis was classified as secondary progressive when an initial relapsing-remitting phase was followed by steady worsening of neurological deficits with or without superimposed bouts $[2,18]$.

PPMS was defined as at least one year of deterioration from disease onset with or without superimposed episodes of worsening $[1,22]$.

Therefore, the number of relapses in the previous two years was determined only for patients with relapsing-remitting and secondary-progressive forms of MS. All patients underwent a clinical neurological examination during every infusion of MTX. Their disability was assessed using Kurtzke's EDSS scale [17]. All patients provided informed consent to having their data saved in the database.

\section{Drug administration and safety profile}

The routine protocol included seven consecutive cycles of MTX - the first five every three months and the last two every six months. For each cycle of treatment, the patient was admitted to the Neurology Department for three consecutive days. On the first and third days, dexamethasone was administered intravenously (iv) at a dose of $8 \mathrm{mg}$. On the second day, the patients received a 30-min infusion of MTX iv at a starting dose of $12 \mathrm{mg} / \mathrm{m}^{2}$, directly preceded by the administration of ondansetron (8 $\mathrm{mg}$ iv/dose) [10]. A separate treatment regimen was used for an induction therapy in aggressive RRMS: this consisted of three cycles of treatment usually at one- or three-monthly intervals [10,23]. Additional courses of steroid (methylprednisolone, $1 \mathrm{~g}$ iv /day for five days) were allowed only for relapses. At the beginning of each treatment cycle, blood and urine samples were taken and echocardiography was performed. The adverse events were assessed on the basis of data obtained from patients either spontaneously or on being questioned, as well as monitoring of laboratory and echocardiographic parameters. In addition, each patient was required to perform a control complete blood count and peripheral blood smear after two weeks of MTX infusion. MTX adverse reactions were divided according to the time from drug administration to their occurrence into either immediate (up to three days after infusion), or early (from day 4 to one month after infusion), or delayed (more than one month after infusion).

\section{MRI assessment}

Magnetic resonance imaging (MRI) exams of brain and cervical spinal cord were carried out each time before the 
first and after the last dose of MTX. All of the studies were performed on a 1.5 Tesla MRI scanner using a standardised protocol. MRI data was analysed by two neuro-radiologists who assessed the number of new gadolinium-enhancing $(\mathrm{Gd}+)$ lesions on postcontrast T1-weighted scans.

\section{Outcome criteria and statistical analysis}

The primary outcome criteria were to compare the efficacy of mitoxantrone in various forms of multiple sclerosis, and to see if there was a new place for the drug in the treatment regimen of this disease. The clinical efficacy of the drug was assessed by comparing EDSS score at baseline to that after the last MTX infusion, as well as the proportion of exacerbationfree patients. The term 'improvement' was used when the median EDSS score was lower at the end of the treatment than at baseline; the term 'stabilisation' was used when the median EDSS score did not change after MTX therapy; and the term 'deterioration' was used when the median EDSS score after treatment was higher than at the beginning. The secondary outcome criteria were decreases in the number of Gd+ lesions on MRI scans after the last MTX infusion compared to the baseline study, and in the frequency and profile of treatment-adverse reactions. The treatment side effects were then correlated with the clinical course of the disease and with gender. An assessment of the above-mentioned outcomes was also performed in patients who prematurely ended MTX therapy.

The differences between the investigated groups were calculated by Student's t-test for parametric data and U MannWhitney test for nonparametric data. Chi-squared test was used to compare proportions in groups. Statistical significance was considered for a $\mathrm{p}$-value $\leq 0.05$. All calculations were performed with a Statistica 12.1 application.

\section{Results}

\section{Clinical characteristics}

The investigated group consisted of 64 women $(64 \%)$ and 36 men $(36 \%)$ with a mean age of $44.3 \pm 10.8$ years. The mean age at disease onset was $34.9 \pm 11.2$ years. The mean disease duration was $9.5 \pm 7.1$ years. The median EDSS score at baseline was 4.5 , range $2-7.36$ of the patients (36\%) were assigned to the RRMS subgroup, 36 to the SPMS subgroup (36\%), and $28(28 \%)$ to the PPMS subgroup with respect to disease course. The mean time to transition to a secondary progressive course of MS was $12.1 \pm 6.4$ years. We did not find significant clinical differences between women and men. The clinical and demographic characteristics of the investigated groups are set out in Table 1. 19 of the patients had used immunomodulatory therapies (IMTs) within the two years preceding MTX infusion: glatiramer acetate - three, natalizumab - one, interferon beta -13 , glatiramer acetate and interferon beta at intervals - two. The median time of immunomodulatory therapy among these patients was two years. Among patients with relapsing-remitting onset of MS, 54 had experienced one or more bouts within the two years preceding mitoxantrone therapy (Tab. 1). We assessed the primary and secondary outcome criteria, as well as the treatment of adverse events in all investigated patients.

\section{Clinical outcomes}

The mean time of treatment was $1.9 \pm 0.6$ years. The mean number of cycles of MTX infusion was $6.8 \pm 1.97$, and the median total dose was $140 \mathrm{mg}$ (range 30-140). 36 patients did not complete the full course of MTX therapy for the following reasons: 16 patients with RRMS were switched to immunomodulatory treatment; 14 patients felt the drug was ineffective; and six patients withdrew due to adverse events. In the investigated group, the median EDSS score at baseline was 4.5 (range 2-7) and remained stable during the treatment period. Comparing the EDSS score at baseline and after the last MTX infusion, we found that disability was worse by at least 0.5 EDSS point in 27 patients, by at least one point in 10 patients, and improved in 31 patients ( $\geq 0.5$ point). The remaining 47 patients had no change in EDSS score during the treatment period. The median time to identify an improvement on the EDSS scale was three months (range 3-18). The mean time to disability progression was $10.5 \pm 3.7$ months. We found statistically significant differences in disability progression with regard to the variant of multiple sclerosis, with the duration of relapsing-onset MS (RRMS/SPMS), and with gender (Tab. 1, 2). Among the patients with confirmed disability progression $\geq 1.0$ EDSS point, there were no RRMS patients; eight patients had PPMS, and the remaining two had SPMS $(\mathrm{p}=0.0004)$. We observed a decrease in the median EDSS score from 4.0 at baseline to 3.5 at the end of MTX infusion in the RRMS subgroup and an increase from 4.5 to 5.25 in the PPMS subgroup. In the subgroup of patients with SPMS, the median EDSS score at baseline was 5.0 and remained stable during the treatment period. We found statistically significant differences in EDSS score between the baseline and the end of MTX infusion in patients who had had at least one exacerbation within the 12 months preceding mitoxantrone therapy (Tab. 2). During the treatment period, one person experienced one relapse, and another two relapses, of multiple sclerosis. Thus, 70 patients with an initial relapsing-remitting course of MS were free of exacerbations.

\section{Assessment of brain MRI activity}

43 patients had at least one gadolinium-enhancing lesion at baseline MRI. Of these, in 30 subjects they were located only in the brain, in eight patients only in the spinal cord, and in the remaining five patients in both locations simultaneously. The median number of baseline Gd+ lesions was 2 (range 1-4). At the beginning of the treatment, T1-Gd+ lesions were found in 24 patients with RRMS, 12 with SPMS, and seven with PPMS ( $p=0.00129)$. At the end of mitoxantrone therapy, gadolinium-enhancing MRI lesions were noted in 
Table 1. Demographic and clinical characteristics of patients

\begin{tabular}{|c|c|c|c|c|}
\hline & General & Women & Men & p-value \\
\hline No. of patients & 100 & 64 & 36 & \\
\hline \multicolumn{5}{|l|}{ Age (years) } \\
\hline Mean (SD) & $44.3(10.8)$ & $44.0(11.6)$ & $44.7(9.5)$ & \multirow[t]{2}{*}{0.7507} \\
\hline Median (range) & $45(20-68)$ & $45(20-68)$ & $43(30-63)$ & \\
\hline \multicolumn{5}{|l|}{ Age at disease onset (years) } \\
\hline Mean (SD) & $34.9(11.2)$ & $34.5(11.4)$ & $35.811 .0)$ & \multirow[t]{2}{*}{0.5738} \\
\hline Median (range) & $34(13-61)$ & $32.5(13-58)$ & $35(17-61)$ & \\
\hline \multicolumn{5}{|l|}{ Disease course (at the beginning of treatment) } \\
\hline Relapsing-remitting, n (\%) & $36(36)$ & $26(40.6)$ & $10(27.8)$ & \multirow[t]{3}{*}{0.1695} \\
\hline Secondary progressive, $\mathrm{n}(\%)$ & $36(36)$ & $24(37.5)$ & $12(33.3)$ & \\
\hline Primary progressive, $\mathrm{n}(\%)$ & $28(28)$ & $14(21.9)$ & $14(38.9)$ & \\
\hline \multicolumn{5}{|l|}{ Disease duration (years) } \\
\hline Mean (SD) & $9.5(7.1)$ & $9.8(7.2)$ & $8.9(6.8)$ & \multirow[t]{2}{*}{0.6614} \\
\hline Median (range) & $7(1-32)$ & $6.5(1-32)$ & $8(1-24)$ & \\
\hline \multicolumn{5}{|l|}{ Systems involved at onset; $n$ of patients } \\
\hline Sensory & 13 & 11 & 2 & \multirow[t]{8}{*}{0.3466} \\
\hline Motor & 12 & 9 & 3 & \\
\hline Sensory and motor & 2 & 2 & 0 & \\
\hline Optic neuritis & 19 & 10 & 9 & \\
\hline Brainstem & 6 & 4 & 2 & \\
\hline Cerebellar & 17 & 11 & 6 & \\
\hline Spinal & 21 & 10 & 11 & \\
\hline Polysymptomatic & 10 & 7 & 3 & \\
\hline \multicolumn{5}{|l|}{ EDSS score at baseline } \\
\hline Mean (SD) & $4.6(1.1)$ & $4.7(1.1)$ & $4.6(1.1)$ & \multirow[t]{2}{*}{0.9748} \\
\hline Median (range) & $4.5(2-7)$ & $4.5(2-6.5)$ & $4.5(2.5-7)$ & \\
\hline \multicolumn{5}{|l|}{ Relapses before MTX infusion } \\
\hline 12 months & 44 & 32 & 12 & \multirow[t]{2}{*}{0.7972} \\
\hline 24 months & 54 & 38 & 16 & \\
\hline \multicolumn{5}{|l|}{ Change in EDSS score after MTX infusions } \\
\hline Improvement & 31 & 25 & 6 & \multirow[t]{3}{*}{0.0450} \\
\hline Stabilisation & 42 & 22 & 20 & \\
\hline Deterioration & 27 & 17 & 10 & \\
\hline \multicolumn{5}{|l|}{ MTX adverse reactions } \\
\hline \multicolumn{5}{|l|}{ a) immediate } \\
\hline Nausea & 29 & 21 & 8 & 0.2626 \\
\hline Vomiting & 13 & 12 & 1 & 0.0226 \\
\hline \multicolumn{5}{|l|}{ b) early } \\
\hline Leucopenia $(<3,000 / \mathrm{mm} \rrbracket)$ & 21 & 17 & 4 & 0.0686 \\
\hline Urinary and upper respiratory tract infection & 14 & 9 & 5 & 0.9808 \\
\hline Alopecia & 12 & 12 & 0 & 0.0056 \\
\hline \multicolumn{5}{|l|}{ c) delayed } \\
\hline Decreased LVEF & 7 & 3 & 4 & 0.2269 \\
\hline
\end{tabular}


Table 2. Change in EDSS score after MTX infusions

\begin{tabular}{|c|c|c|c|c|}
\hline \multirow[t]{2}{*}{ Type of parameter } & \multicolumn{3}{|c|}{ Change in EDSS score } & \multirow[b]{2}{*}{ p-value } \\
\hline & Improvement & Stabilisation & Deterioration & \\
\hline \multicolumn{5}{|l|}{ Clinical course of MS } \\
\hline RRMS & 22 & 11 & 3 & \multirow[t]{3}{*}{$<0.0001$} \\
\hline SPMS & 9 & 21 & 6 & \\
\hline PPMS & 0 & 10 & 18 & \\
\hline \multicolumn{5}{|l|}{ Disease duration } \\
\hline Relapsing-remitting onset MS & 12 & 2 & 2 & \multirow{4}{*}{0.0017} \\
\hline$<5$ years & 7 & 9 & 6 & \\
\hline $5-10$ years & 12 & 21 & 1 & \\
\hline$>10$ years & & & 15 & \\
\hline \multicolumn{5}{|l|}{ PPMS } \\
\hline$<5$ years & 0 & 7 & & \multirow[t]{3}{*}{0.3705} \\
\hline $5-10$ years & 0 & 3 & 2 & \\
\hline$>10$ years & 0 & 0 & 1 & \\
\hline \multicolumn{5}{|c|}{ Relapses in the 12 months prior to MTX } \\
\hline No & 6 & 19 & 3 & \multirow[t]{2}{*}{0.0046} \\
\hline$\geq 1$ & 25 & 13 & 6 & \\
\hline \multicolumn{5}{|c|}{ Relapses in the 24 months prior to MTX } \\
\hline No & 6 & 10 & 2 & \multirow[t]{2}{*}{0.5405} \\
\hline$\geq 1$ & 25 & 22 & 7 & \\
\hline \multicolumn{5}{|l|}{ T1-Gd+ lesions at baseline MRI } \\
\hline No & 9 & 28 & 20 & \multirow[t]{2}{*}{0.0006} \\
\hline$\geq 1$ & 22 & 14 & 7 & \\
\hline
\end{tabular}

six patients. In four of them, these lesions were located in the brain, and in two in the spinal cord. We found a statistically significant improvement in EDSS score between the beginning and the end of MTX infusion in patients who had at least one gadolinium-enhancing lesion at baseline MRI ( $p=0.00046)$ (Tab. 2). The median number of baseline $\mathrm{Gd}+$ lesions was 1 in the subgroup of patients who experienced disability reduction after MTX therapy, and 0 in the subgroup of patients with stabilisation or progression of disability at the end of this treatment $(\mathrm{p}=0.0038)$.

\section{Adverse events}

No patient developed acute myeloid leukemia. There was no congestive heart failure. Seven patients developed asymptomatic left ventricular ejection fraction decreased to less than $50 \%$. Nine women experienced dysmenorrhea. There were no serious infections, 10 patients experienced mild urinary tract infections, and four patients experienced upper respiratory tract infections that resolved with antibiotic therapy. Alopecia was transient and minor for 12 women, and no patient developed moderate or severe alopecia. We found a statistically significant difference in the development of vomiting and alopecia between men and women (Tab. 1). There were no statistically significant correlations between the clinical MS variant and the incidence of MTX adverse events.

\section{Discussion}

The efficacy and safety profile of mitoxantrone have been evaluated in many previous studies, and these became the basis for the approval of this treatment by the FDA. However, most of these trials did not assess a comparative analysis of the differences in response to treatment between patients with RRMS and SPMS, and none of them focused on patients on the borderline of RRMS and SPMS [10, 24-26].

In the presented study, we performed a post hoc analysis of the influence of MTX on the clinical and neuroradiological activity of different MS variants. Regarding the primary outcomes, we demonstrated the efficacy of mitoxantrone in the reduction of disability progression depending on the variant and stage of the disease. We found an improvement in the EDSS score in $61 \%$ of patients with RRMS and in $25 \%$ of patients with SPMS at the end of MTX therapy. The proportion of disability progression-free patients during the period 
of evaluation was $92 \%$ in the RRMS subgroup and $83 \%$ in the SPMS subgroup. It is noteworthy that the mean time to achieve an improvement in EDSS score was three months. Taking into account all patients with relapsing-onset MS, MTX prevented disability progression in $87.5 \%$ of them, and the efficacy of the drug was significantly higher in subjects with a shorter disease duration. In this subgroup, $97 \%$ of patients were free of relapses during the treatment period. Esposito et al. compared the efficacy of MTX in 79 patients with RRMS and 210 patients with SPMS [27]. The authors found $94.9 \%$ of RRMS patients free from disability progression during the treatment period. The mean EDSS score improved in the RRMS subgroup from 3.0 to 2.6 after treatment with MTX. The improvement was usually observed during the first trimester of treatment, which makes the results of this Italian study consistent with our data. Compared to our study, Esposito et al. obtained a lower proportion of patients with SPMS (71\%) free from disability accumulation, and a lower proportion of relapsing-onset MS patients (50\%) free of bouts during the treatment period. This discrepancy might be explained by the lower MTX cumulative dose $\left(61 \mathrm{mg} / \mathrm{m}^{2}\right)$ used by the authors.

Many studies have confirmed the efficacy of mitoxantrone in the treatment of RRMS patients with high disease activity. In these cases, mitoxantrone reduced the annualised relapse rate, the disability progression, and the number of demyelination lesions with contrast enhancement [13, 28-30]. These figures are comparable to our findings in the subgroup of patients with RRMS. In our study, among all relapsing-onset MS patients $26 \%$ were exposed to immunomodulatory drugs during the two years preceding MTX infusions. Termination of IMTs was due to its lack of efficacy in the course of high disease activity or the initial phase of conversion from RRMS to SPMS. For this reason, MTX remained the most beneficial therapeutic option for most of these patients. The identification and treatment planning for patients with RRMS who started conversion to SPMS was a particular challenge. In the natural course of MS, the conversion from RRMS to SPMS is observed between 5.8 and 19.1 years from disease onset. This timespan may be a consequence of the fear of recognising the next stage of the disease and, at the same time, the lack of efficacy of the IMTs used. Furthermore, it may result from the application of differing diagnostic criteria. Difficulties in identifying the early stages of SPMS can lead to diagnostic uncertainty and inadequate treatment. Sand et al. determined the average delay in diagnosis of SPMS to be $2.9 \pm 0.8$ years [31]. In our study, the patients were distributed into SPMS according to the Lublin and Reingold criteria [2] in which a steady worsening of neurological symptoms for at least six months, independent of bouts, was a cardinal condition for diagnosis. However, in some of the studies conducted so far, the observation period of the neurological deficit deterioration needed to establish the diagnosis of SPMS was 12 months, and the other proposed criteria included only a 3-month observation assuming a minimum disability greater than or equal to
4 EDSS points [32]. For this reason, the baseline EDSS score of 4 in the RRMS subgroup of our patients could be an additional argument supporting the beginning of the transition to SPMS.

Thus, our results may indicate that there is a new place for mitoxantrone in the treatment of MS for patients on the borderline of RRMS and SPMS. The multicentre, randomised, double-blind, placebo-controlled study published by Hartung et al. revealed the efficacy of $12 \mathrm{mg} / \mathrm{m}^{2}$ of mitoxantrone in the treatment of worsening RRMS and SPMS patients compared to placebo [10]. The authors obtained a reduction in the progress of disability and the annualised relapse rate by $64 \%$ and $60 \%$ respectively in the mitoxantrone group. However, this study did not assess the efficacy of MTX in patients who are on the borderline of RRMS and SPMS.

Our study found an $86 \%$ decrease in the proportion of patients with gadolinium-enhancing lesions after MTX infusions, which was in line with the results presented by Edan et al. and Le Page et al. [24, 28]. Additionally, we showed that both the presence of at least one Gd+ lesion at baseline MRI, and at least one relapse within the 12 months preceding MTX therapy, significantly improved EDSS score after treatment. In our group, no PPMS patient was free of disability progression after MTX therapy, which confirms previous findings of ineffectiveness of the drug in this MS variant [33]. However, it should be noted that in $35.7 \%$ of patients in this subgroup, EDSS score remained stable during the treatment. In our study, the highest percentage of men was in the PPMS subgroup, and the highest percentage of women was in the RRMS subgroup, which could explain the worse clinical response of men than women to mitoxantrone.

In the investigated group, mitoxantrone was generally well tolerated. There were no life-threatening adverse events of MTX during the treatment period. Nausea was the most frequently reported side effect. An asymptomatic decreased left ventricular ejection fraction to less than $50 \%$ was found in $7 \%$ of patients, which was comparable to the results obtained in the study by Esposito et al. [27]. Leukopenia was reported in $21 \%$ of patients and was transient in each case.

A valuable finding of our study is the occurrence of some adverse effects (vomiting and alopecia) found significantly more often in women than in men. Due to the lack of data on this issue in the literature, it was impossible to compare these results with those of other studies. In our study, all reported short-term adverse events of MTX were less frequent than in previous reports [10, 24, 34-36]. Unfortunately, we have not conducted a long-term assessment of the MTX safety profile in our group, which is one of the limitations of the study. For this reason, we were unable to monitor the left-ventricular ejection fraction up to five years after MTX therapy cessation, as recommended by EMA. So, the percentage of patients who have experienced cardiotoxic effects of the drug may be underestimated. Furthermore, the number of subjects using IMTs prior to MTX therapy was small, mainly due to administrative reasons and the time restrictions regarding this treatment in 
Poland until 2014 [37]. Therefore, mitoxantrone became the first line treatment in $74 \%$ of subjects with relapsing-onset MS. Another limitation was the lack of MRI assessment for new or enlarging T2 lesions. However, the main limitation of our study remains its retrospective nature.

\section{Clinical implications/future directions}

The results of our study suggest that mitoxantrone is a valuable therapeutic option for patients with highly active MS in whom other treatments are ineffective or unavailable. The use of MTX can be justified in patients with SPMS, especially in the context of the unmet therapeutic need for this MS variant. The drug is useless in patients with PPMS.

Finally, our findings indicate that mitoxantrone may be considered in patients at the initial stages of conversion from RRMS to SPMS in the case of ineffectiveness of the available IMTs or contraindications for their use. To the best of our knowledge, this is the first study to have indicated a place for mitoxantrone in the treatment of patients who are on the borderline of RRMS and SPMS.

Funding: This publication was prepared without any external source of funding.

Conflicts of interest: None

\section{References}

1. Weinshenker BG, Bass B, Rice GP, et al. The natural history of multiple sclerosis: a geographically based study. I. Clinical course and disability. Brain. 1989; 112 ( Pt 1): 133-146, doi: 10.1093/brain/112.1.133, indexed in Pubmed: 2917275.

2. Lublin FD, Reingold SC. Defining the clinical course of multiple sclerosis: results of an international survey. National Multiple Sclerosis Society (USA) Advisory Committee on Clinical Trials of New Agents in Multiple Sclerosis. Neurology. 1996; 46(4): 907-911, doi: 10.1212/ wnl.46.4.907, indexed in Pubmed: 8780061.

3. Confavreux $\mathrm{C}$, Vukusic $\mathrm{S}$. Natural history of multiple sclerosis: a unifying concept. Brain. 2006; 129(Pt 3): 606-616, doi: 10.1093/brain/ awl007, indexed in Pubmed: 16415308.

4. Montalban X, Gold R, Thompson AJ, et al. ECTRIMS/EAN guideline on the pharmacological treatment of people with multiple sclerosis. Eur J Neurol. 2018; 25(2): 215-237, doi: 10.1111/ene.13536, indexed in Pubmed: 29352526.

5. Cottrell DA, Kremenchutzky M, Rice GP, et al. The natural history of multiple sclerosis: a geographically based study. 5 . The clinical features and natural history of primary progressive multiple sclerosis. Brain. 1999; 122 ( Pt 4): 625-639, doi: 10.1093/brain/122.4.625, indexed in Pubmed: 10219776.

6. Miller D, Leary S. Primary-progressive multiple sclerosis. The Lancet Neurology. 2007; 6(10): 903-912, doi: 10.1016/s14744422(07)70243-0.

7. EMD Serono, Inc. Novantrone (mitoxantrone) for injection concentrate [product monograph]. http://www.novantrone.com/assets/pdf/novantrone_prescribing_info. pdf. (October 20, 2007.).
8. Fidler JM, DeJoy SQ, Smith FR, et al. Selective immunomodulation by the antineoplastic agent mitoxantrone. II. Nonspecific adherent suppressor cells derived from mitoxantrone-treated mice. J Immunol. 1986; 136(8): 2747-2754, indexed in Pubmed: 2937837.

9. Neuhaus 0 , Kieseier BC, Hartung HP. Mechanisms of mitoxantrone in multiple sclerosis--what is known? J Neurol Sci. 2004; 223(1): 25-27, doi: 10.1016/j.jns.2004.04.015, indexed in Pubmed: 15261556.

10. Hartung HP, Gonsette R, König N, et al. Mitoxantrone in Multiple Sclerosis Study Group (MIMS). Mitoxantrone in progressive multiple sclerosis: a placebo-controlled, double-blind, randomised, multicentre trial. Lancet. 2002; 360(9350): 2018-2025, doi: 10.1016/S0140-6736(02)12023-X, indexed in Pubmed: 12504397.

11. Bhatia R, Singh N. Can We Treat Secondary Progressive Multiple Sclerosis Now? Ann Indian Acad Neurol. 2019; 22(2): 131-136, doi: 10.4103/aian.AIAN_345_18, indexed in Pubmed: 31007422.

12. https://www.ema.europa.eu/en/documents/referral/novantronearticle-30-referral-annex-iii_en.pdf.

13. Buttmann $M$. Where mitoxantrone for multiple sclerosis is still valuable in 2018. Eur J Neurol. 2018; 25(12): 1400-1401, doi: 10.1111/ ene.13787, indexed in Pubmed: 30133060.

14. Giovannoni G. Disease-modifying treatments for early and advanced multiple sclerosis: a new treatment paradigm. Curr Opin Neurol. 2018; 31(3): 233-243, doi: 10.1097/WC0.0000000000000561, indexed in Pubmed: 29634596.

15. Mather FJ, Simon RM, Clark GM, et al. Cardiotoxicity in patients treated with mitoxantrone: Southwest Oncology Group phase II studies. Cancer Treat Rep. 1987; 71(6): 609-613, indexed in Pubmed: 3581099.

16. Goodin DS, Arnason BG, Coyle PK, et al. Therapeutics and Technology Assessment Subcommittee of the American Academy of Neurology. The use of mitoxantrone (Novantrone) for the treatment of multiple sclerosis: report of the Therapeutics and Technology Assessment Subcommittee of the American Academy of Neurology. Neurology. 2003; 61(10): 1332-1338, doi: 10.1212/01.wnl.0000095425.84407.39, indexed in Pubmed: 14638950.

17. Kurtzke JF. Rating neurologic impairment in multiple sclerosis: an expanded disability status scale (EDSS). Neurology. 1983; 33(11): 1444-1452, doi: 10.1212/wnl.33.11.1444, indexed in Pubmed: 6685237.

18. Poser CM, Paty DW, Scheinberg L, et al. New diagnostic criteria for multiple sclerosis: guidelines for research protocols. Ann Neurol. 1983; 13(3): 227-231, doi: 10.1002/ana.410130302, indexed in Pubmed: 6847134.

19. McDonald WI, Compston A, Edan G, et al. Recommended diagnostic criteria for multiple sclerosis: guidelines from the International Panel on the diagnosis of multiple sclerosis. Ann Neurol. 2001; 50(1): 121-127, doi: 10.1002/ana.1032, indexed in Pubmed: 11456302.

20. Polman $\mathrm{CH}$, Reingold SC, Edan G, et al. Diagnostic criteria for multiple sclerosis: 2005 revisions to the "McDonald Criteria". Ann Neurol. 2005; 58(6): 840-846, doi: 10.1002/ana.20703, indexed in Pubmed: 16283615.

21. Polman $\mathrm{CH}$, Reingold SC, Banwell B, et al. Diagnostic criteria for multiple sclerosis: 2010 revisions to the McDonald criteria. Ann Neurol. 2011; 69(2): 292-302, doi: 10.1002/ana.22366, indexed in Pubmed: 21387374.

22. Scalfari A, Neuhaus A, Degenhardt A, et al. The natural history of multiple sclerosis: a geographically based study 10: relapses and longterm disability. Brain. 2010; 133(Pt 7): 1914-1929, doi: 10.1093/ brain/awq118, indexed in Pubmed: 20534650. 
23. Chartier N, Epstein J, Soudant M, et al. Clinical follow-up of 411 patients with relapsing and progressive multiple sclerosis 10 years after discontinuing mitoxantrone treatment: a real-life cohort study. Eur J Neurol. 2018; 25(12): 1439-1445, doi: 10.1111/ene.13748, indexed in Pubmed: 29996003.

24. Edan G, Miller D, Clanet M, et al. Therapeutic effect of mitoxantrone combined with methylprednisolone in multiple sclerosis: a randomised multicentre study of active disease using MRI and clinical criteria. J Neurol Neurosurg Psychiatry. 1997; 62(2): 112-118, doi: 10.1136/jnnp.62.2.112, indexed in Pubmed: 9048709.

25. Debouverie M, Taillandier L, Pittion-Vouyovitch S, et al. Clinical follow-up of 304 patients with multiple sclerosis three years after mitoxantrone treatment. Mult Scler. 2007; 13(5): 626-631, doi: 10.1177/1352458506072543, indexed in Pubmed: 17548442.

26. Buttinelli $\mathrm{C}$, Clemenzi $\mathrm{A}$, Borriello $\mathrm{G}$, et al. Mitoxantrone treatment in multiple sclerosis: a 5-year clinical and MRI follow-up. Eur J Neurol. 2007; 14(11): 1281-1287, doi: 10.1111/j.1468-1331.2007.01969.x, indexed in Pubmed: 17956449.

27. Esposito F, Radaelli M, Martinelli V, et al. Comparative study of mitoxantrone efficacy profile in patients with relapsing-remitting and secondary progressive multiple sclerosis. Mult Scler. 2010; 16(12): 1490-1499, doi: 10.1177/1352458510379613, indexed in Pubmed: 20810516.

28. Le Page E, Leray E, Taurin G, et al. Mitoxantrone as induction treatment in aggressive relapsing remitting multiple sclerosis: treatment response factors in a 5 year follow-up observational study of 100 consecutive patients. J Neurol Neurosurg Psychiatry. 2008; 79(1): 52-56, doi: 10.1136/jnnp.2007.124958, indexed in Pubmed: 17846110.

29. Cocco E, Marchi P, Sardu C, et al. Mitoxantrone treatment in patients with early relapsing-remitting multiple sclerosis. Mult Scler. 2007; 13(8): 975-980, doi: 10.1177/1352458507077621, indexed in Pubmed: 17468439.
30. Edan G, Comi G, Le Page E, et al. French-Italian Mitoxantrone Interferon-beta-1b Trial Group. Mitoxantrone prior to interferon beta$1 \mathrm{~b}$ in aggressive relapsing multiple sclerosis: a 3-year randomised trial. J Neurol Neurosurg Psychiatry. 2011; 82(12): 1344-1350, doi: 10.1136/jnnp.2010.229724, indexed in Pubmed: 21436229.

31. Katz Sand I, Krieger S, Farrell C, et al. Diagnostic uncertainty during the transition to secondary progressive multiple sclerosis. Mult Scler. 2014; 20(12): 1654-1657, doi: 10.1177/1352458514521517, indexed in Pubmed: 24493475.

32. Lorscheider J, Buzzard K, Jokubaitis V, et al. MSBase Study Group. Defining secondary progressive multiple sclerosis. Brain. 2016; 139(Pt 9): 2395-2405, doi: 10.1093/brain/aww173, indexed in Pubmed: 27401521.

33. Filippini G, Del Giovane C, Vacchi L, et al. Immunomodulators and immunosuppressants for multiple sclerosis: a network metaanalysis. Cochrane Database Syst Rev. 2013(6): CD008933, doi: 10.1002/14651858.CD008933.pub2, indexed in Pubmed: 23744561.

34. Millefiorini E, Gasperini C, Pozzilli C, et al. Randomized placebocontrolled trial of mitoxantrone in relapsing-remitting multiple sclerosis: 24-month clinical and MRI outcome. J Neurol. 1997; 244(3): 153-159, doi: 10.1007/s004150050066, indexed in Pubmed: 9050955.

35. van de Wyngaert FA, Beguin C, D'Hooghe MB, et al. A double-blind clinical trial of mitoxantrone versus methylprednisolone in relapsing, secondary progressive multiple sclerosis. Acta Neurol Belg. 2001; 101(4): 210-216, indexed in Pubmed: 11851027.

36. Martinelli Boneschi F, Rovaris M, Capra R, et al. Mitoxantrone for multiple sclerosis. Cochrane Database Syst Rev. 2005(4): CD002127, doi: 10.1002/14651858.CD002127.pub2, indexed in Pubmed: 16235298.

37. http://www.mz.gov.pl/leki/refundacja/programy-lekowe. 